\title{
Internal structure and spatial dimensions of whistler wave regions in the magnetopause boundary layer
}

\author{
G. Stenberg ${ }^{1,2}$, T. Oscarsson ${ }^{1, \dagger}$, M. André ${ }^{2}$, A. Vaivads $^{2}$, M. Backrud-Ivgren ${ }^{2,3}$, Y. Khotyaintsev $^{2}$, L. Rosenqvist ${ }^{2}$, \\ F. Sahraoui ${ }^{4}$, N. Cornilleau-Wehrlin ${ }^{4}$, A. Fazakerley ${ }^{5}$, R. Lundin ${ }^{6}$, and P. M. E. Décréau ${ }^{7}$ \\ ${ }^{1}$ Department of Physics, Umeå University, Umeå, Sweden \\ ${ }^{2}$ Swedish Institute of Space Physics, Uppsala, Sweden \\ ${ }^{3}$ Division of Plasma Physics, Alfvén Laboratory, Royal Institute of Technology, Stockholm, Sweden \\ ${ }^{4}$ CETP/UVSQ, Vélizy, France \\ ${ }^{5}$ MSSL, University College, London, UK \\ ${ }^{6}$ Swedish Institute of Space Physics, Kiruna, Sweden \\ ${ }^{7}$ LPCE et Université d'Orléans, France \\ $\dagger$ deceased
}

Received: 20 May 2007 - Revised: 27 October 2007 - Accepted: 5 November 2007 - Published: 29 November 2007

\begin{abstract}
We use whistler waves observed close to the magnetopause as an instrument to investigate the internal structure of the magnetopause-magnetosheath boundary layer. We find that this region is characterized by tube-like structures with dimensions less than or comparable with an ion inertial length in the direction perpendicular to the ambient magnetic field. The tubes are revealed as they constitute regions where whistler waves are generated and propagate. We believe that the region containing tube-like structures extend several Earth radii along the magnetopause in the boundary layer. Within the presumed wave generating regions we find current structures moving at the whistler wave group velocity in the same direction as the waves.
\end{abstract}

Keywords. Magnetospheric physics (Magnetopause, cusp and boundary layers; Plasma waves and instabilities) - Space plasma physics (Wave-particle interactions)

\section{Introduction}

The magnetopause constitutes the outer frontier defense of the magnetosphere. The border is not completely closed but the entry of the solar wind energy is restricted and regulated by the so-called reconnection process (Dungey, 1961; Paschmann, 1979). Ever since the magnetopause first was suggested to exist (Chapman and Ferraro, 1931) and even more since the first in situ observations where made (e.g. Sonett et al., 1959), there has been a huge interest in revealing its secrets. Numerous studies are devoted to describe, an-

Correspondence to: G. Stenberg

(gabriella@irfu.se) alyze and model the structure and dynamics of this complex boundary.

Lately small-scale structures have received increased attention. The reconnection sites (diffusion regions), where the ion and electron motions are decoupled and the conversion of magnetic energy to particle energy takes place, are the heart of reconnection. Today, computer simulations and spacecraft observations are beginning to resolve the fine-structure involved (e.g. Vaivads et al., 2004a; Onofri et al., 2004; Retinò et al., 2006 and Mozer, 2005). Multi-spacecraft missions, such as Cluster (Escoubet et al., 1997; Escoubet et al., 2001), offer the opportunity to resolve the spatial-temporal ambiguity associated with single spacecraft measurements and allow us to determine the scale lengths of spatial structures.

Fascinating micro-physics are not only restricted to the reconnection sites. Thin electron-scale layers presumably associated with the separatrices are reported far away from the diffusion regions (e.g. André et al., 2004; Vaivads et al., 2004b and Retinò et al., 2006). Stenberg et al. (2005) show that whistler mode waves observed in the boundary layer on the magnetospheric side of the magnetopause are generated in thin (electron-scale) sheets. Furthermore, they suggest that although the sheets of whistler waves are observed thousands of kilometers from the magnetopause, they are still directly related to the diffusion region. As an inherent property of the near-magnetopause region, the thin layers with whistler mode waves and other small-scale structures may be the key to understanding the larger scale phenomena. They may also provide means of monitoring the micro-physics at the reconnection site at large distances.

Whistler-mode waves have been observed by a number of spacecraft in the outer parts of of the magnetosphere. Electromagnetic waves in a broad frequency range are reported

Published by Copernicus Publications on behalf of the European Geosciences Union. 


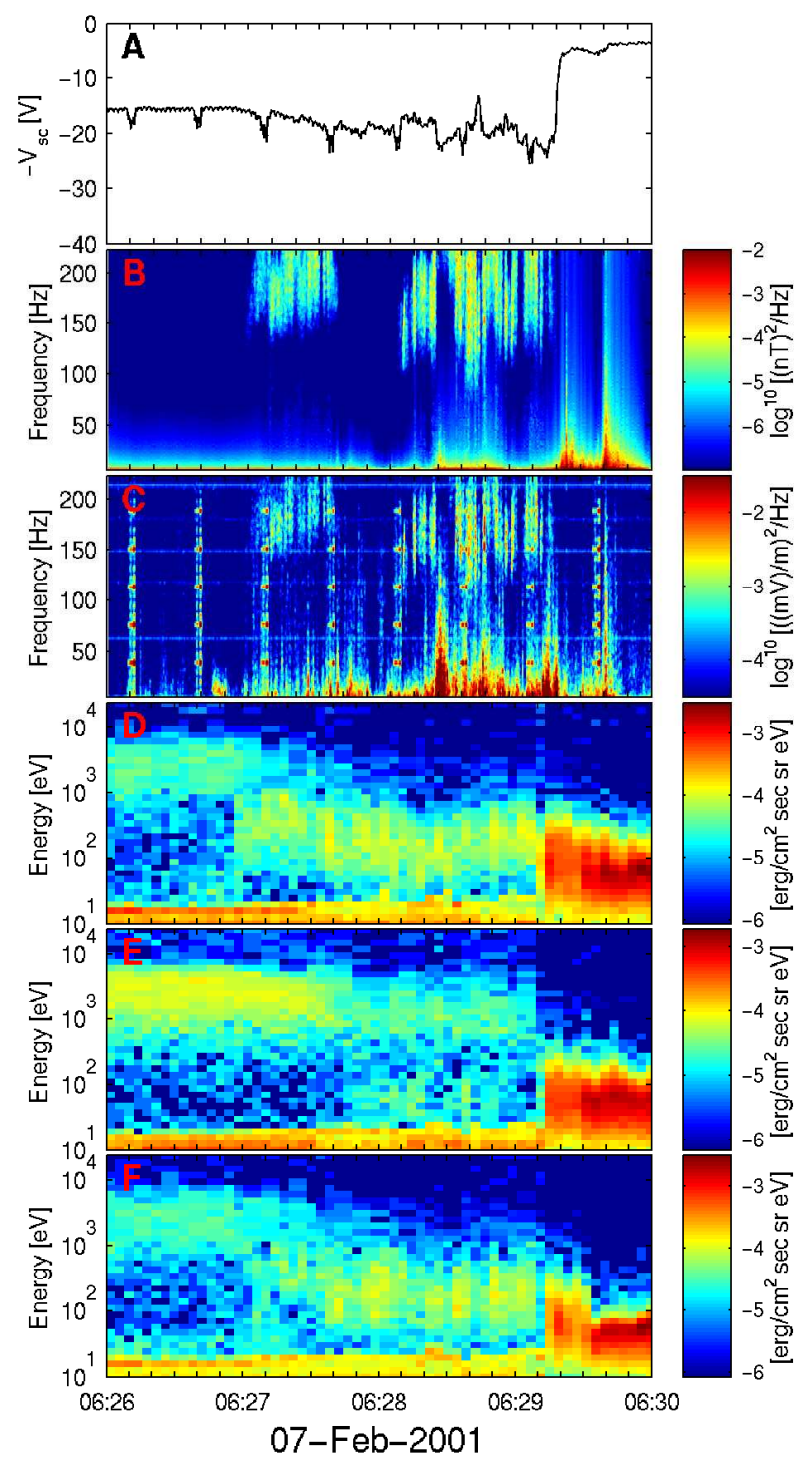

Fig. 1. The top panel shows the probe-to-spacecraft potential, which can be used to estimate the density. The increase in density associated with the magnetopause crossing in clearly seen at 06:29:10 UT. Frequency-time spectrograms of the electric and magnetic wave fields (from EFW and STAFF-SC) are presented in panels (B) and (C). The artificial looking regularly spaced signatures in panel $\mathrm{C}$ as well as the regularly spaced small dips in panel (A) are due to the active mode of the WHISPER instrument. Electron flux versus energy and time (from PEACE) for pitch angles $0^{\circ}, 90^{\circ}$ and $180^{\circ}$ are displayed in the three bottom panels. During the two minutes of intense wave activity preceding the magnetopause crossing there is a mixture of cold $(\sim 100 \mathrm{eV})$ magnetosheath electrons and hot $(\sim 1 \mathrm{keV})$ magnetospheric electrons. All data are from Cluster 2.

from regions close to the magnetopause, in the outer cusp or in association with the bowshock by, e.g., Smith et al. (1967) (OGO-1); Olson et al. (1969) (OGO-3); Rodriguez and Gurnett (1975) (Imp-6); Tsurutani and Smith (1977) (OGO-5);
Gurnett et al., 1979 (ISEE 1 and 2); LaBelle and Treumann (1988) (AMPTE/IRM); Zhang et al. (1998) (Geotail); Pickett et al. (1999) (Polar) and Maksimovic et al. (2001) (Cluster).

In this paper we examine the whistler wave-sheets reported by Stenberg et al. (2005) in closer detail. In particular, we determine the extension of these sheets in all three spatial dimensions and we investigate the internal structure of the presumed wave generating regions. We reveal that the whistler wave source regions are characterized by current structures moving at the whistler wave group velocity in the same direction as the waves themselves. We estimate the size (perpendicular to the background magnetic field) of these structures to be about or less than $20 \mathrm{~km}$, which corresponds to about 6 electron inertial lengths $\left(c / \omega_{p e}\right)$. Simultaneously, our analysis suggests that the whistler wave emitting regions extend several Earth radii along the magnetic field lines.

\section{Data}

The four formation-flying Cluster spacecraft recorded the data used in this study. The satellite orbits are polar with a perigee of 4 Earth radii $\left(R_{E}\right)$ and an apogee of $19.6 R_{E}$. Close to the magnetopause the satellites form almost a tetrahedron. The spacecraft separation, which changes during the mission is $600 \mathrm{~km}$ and $100 \mathrm{~km}$, respectively, for the two events considered below. The spacecraft are spin-stabilized with a spin period of $4 \mathrm{~s}$ and the instrumentation is identical on all four of them (Escoubet et al., 1997; Escoubet et al., 2001).

Data from six of the Cluster instruments are used in this study. The electric field experiment (EFW) (Gustafsson et al., 1997) and the search coil magnetometer (STAFFSC) (Cornilleau-Wehrlin et al., 1997; Cornilleau-Wehrlin et al., 2003) provide time series wave field data. STAFF-SC measures three orthogonal magnetic wave field components, while EFW records two orthogonal electric field components in the satellite spin plane. Both instruments are run in burst mode, i.e., with a sampling rate of 450 samples/second, during both the events considered. The wave field signals are low pass filtered with a cutoff frequency of $180 \mathrm{~Hz}$. We still choose to show data in all the spectrograms up to the Nyquist frequency $(225 \mathrm{~Hz})$, even if there is a small effect of the filter on both the wave amplitude and the phase above $180 \mathrm{~Hz}$.

In addition, the STAFF Spectrum Analyser (STAFF-SA) gives electric and magnetic wave field spectral data in the frequency range $64 \mathrm{~Hz}-4 \mathrm{kHz}$. The WHISPER instrument (Décréau et al., 1997) captures electric wave field emissions in the frequency range $4-80 \mathrm{kHz}$, and provides a way of determining the plasma density from observations of the electron plasma frequency.

The particle environment is investigated using the electron experiment PEACE (Johnstone et al., 1997) and the ion instrument CIS/CODIF (Rème et al., 1997). Both instruments 
can measure a 3-D distribution each spin period. CIS data are also used to estimate the plasma drift velocity.

We also use high resolution data (67.2 vectors/s) from the fluxgate magnetometer (FGM) (Balogh et al., 1997) to transform magnetic wave field data into a background magnetic field oriented coordinate system.

\section{Boundary layer whistlers}

An outbound magnetopause crossing is generally preceded by a passage through a boundary layer, presenting plasma populations of different origins (Eastman et al., 1976). The nature of the boundary layer depends on the latitude and the magnetic local time as well as solar wind and interplanetary magnetic field conditions (Haerendel et al., 1978).

The scene of action for the events to be discussed in this paper is a several thousand kilometer thick boundary layer characterized by a mixture of magnetospheric and magnetosheath plasma. The thickness of the boundary layer is estimated from the time the spacecraft spend in the layer and the magnetopause velocity. The magnetopause passes the different spacecraft at different times, which allow us to calculate its velocity to about $100 \mathrm{~km} / \mathrm{s}$. With a transit time through the boundary layer of about two minutes we arrive at a thickness of $12000 \mathrm{~km}$. In the case that the magnetopause is flapping back and forth during the two minutes, the thickness is overestimated.

The study is based on two events; the first is introduced in Fig. 1. The observations are made by the Cluster spacecraft close to magnetic noon (13:24 MLT) at fairly high latitude (GSE: $x=4.4 \mathrm{R}_{\mathrm{E}}, y=5.7 \mathrm{R}_{\mathrm{E}}, z=9.1 \mathrm{R}_{\mathrm{E}}$ ) on 7 February 2001 . The top panel presents the negative of the probe-to-spacecraft potential. This quantity varies as the density, where a less negative value (closer to zero in panel A) corresponds to a higher density (Pedersen et al., 2001). An outbound (magnetosphere to magnetosheath) magnetopause crossing is seen as a sharp gradient at 06:29:10 UT, where the density suddenly increases.

The middle panels show the electric and magnetic frequency-time spectrograms revealing substantial electromagnetic wave activity in the frequency range $100-200 \mathrm{~Hz}$ during two minutes prior to the crossing. The electron plasma frequency (from WHISPER) is $7.5 \mathrm{kHz}$ and the background magnetic field strength, $\boldsymbol{B}_{0}$, (from FGM) is about $20 \mathrm{nT}$ (GSE: $B_{0 x}=-14 \mathrm{nT}, B_{0 y}=-14 \mathrm{nT}, B_{0 z}=5 \mathrm{nT}$ ), giving an electron gyrofrequency of $560 \mathrm{~Hz}$. Assuming a protonelectron plasma the lower-hybrid frequency can be estimated to about $13 \mathrm{~Hz}$. Hence, the observed electromagnetic waves are within the whistler mode frequency range, above the lower-hybrid frequency and below both the electron gyrofrequency and the plasma frequency. We note that the emissions show a striped pattern, that is, they seem narrow in time and broad in frequency. The high-frequency part of the emis-
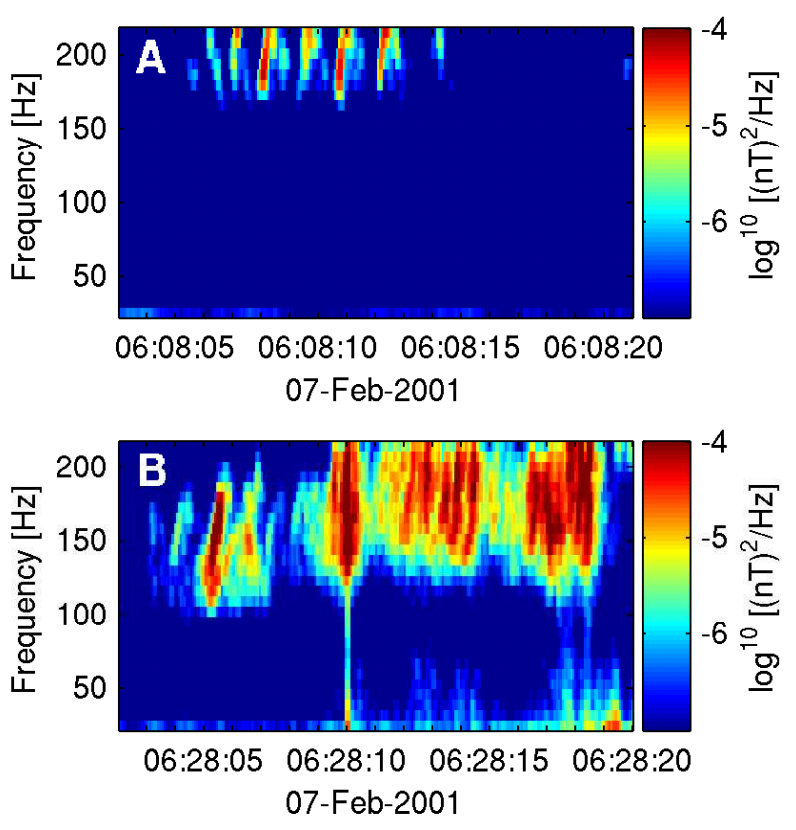

Fig. 2. The near-magnetopause plasma features two different finestructured whistler wave emissions. Panel (A) shows chorus elements observed in an environment of hot magnetospheric plasma only. The striped whistlers in panel (B) are recorded in the boundary layer just inside the magnetopause, where both magnetospheric and colder magnetosheath plasma is present. The data are from STAFF-SC on Cluster 2.

sions are not captured by the time series wave field data, but STAFF-SA shows that the emissions extend up to $400 \mathrm{~Hz}$.

The bottom panels expose the characteristics of the boundary layer: hot $(\sim 1 \mathrm{keV})$ magnetospheric electrons mixed with colder $(\sim 100 \mathrm{eV})$ electrons originating from the magnetosheath. The three panels display electron flux versus energy and time for the pitch angles $0^{\circ}, 90^{\circ}$ and $180^{\circ}$.

The second event we consider is an outbound magnetopause crossing, recorded on 2 March 2002. This event is extensively presented in Stenberg et al. (2005), and at first sight very similar to the event just discussed; waves in the whistler-mode frequency range are detected within a boundary region on the magnetospheric side of the magnetopause boundary. The particle data reveal a mixture of magnetospheric and magnetosheath plasma. The reader is referred to Stenberg et al. (2005) for details and figures corresponding to Fig. 1.

\section{Comparing boundary layer whistlers and chorus}

Approaching the magnetopause we observe a change not only in the the particle distributions but also in the character of the wave emissions. The structure of the waves reveal the entry into the boundary layer. For example, fine-structured whistler waves are intermittently observed for at least half 

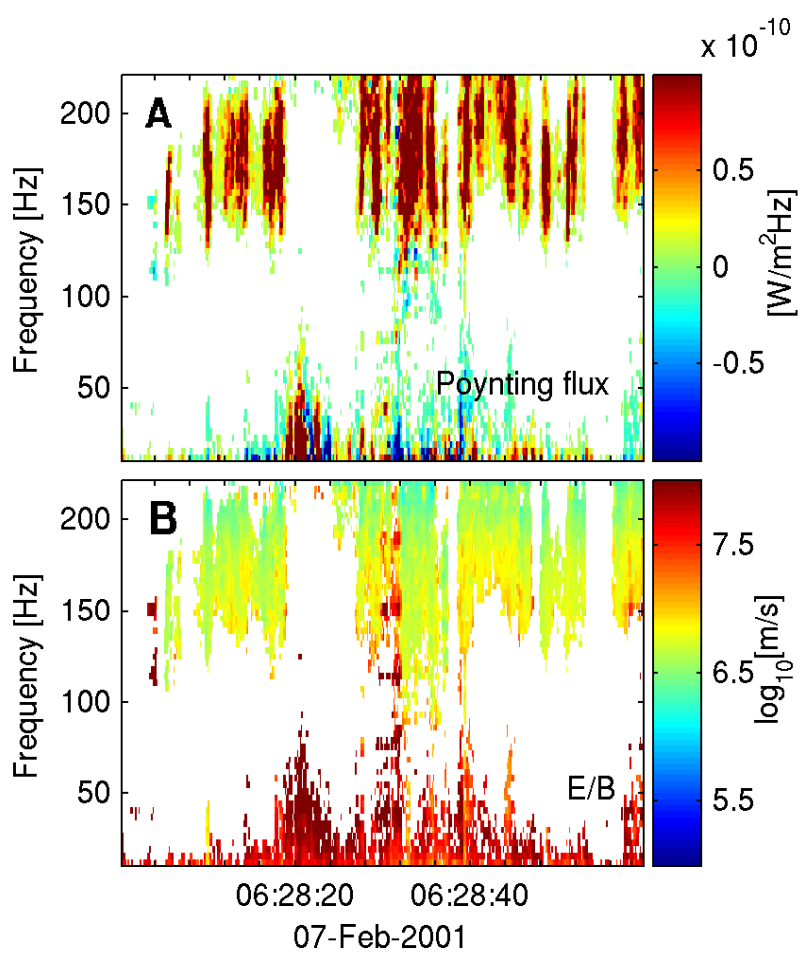

Fig. 3. Panel (A) presents the computed parallel Poynting flux. The whistler mode waves $(>100 \mathrm{~Hz})$ all propagate parallel to the ambient field. Panel (B) shows E/B, which is an estimate of the phase velocity. A phase velocity of about $10^{7} \mathrm{~m} / \mathrm{s}$ corresponds to a wavelength of $100 \mathrm{~km}$ at $100 \mathrm{~Hz}$. EFW and STAFF-SC data from Cluster 2 are used.

an hour preceding the magnetopause crossing on 7 February 2001, this is, long before the spacecraft enter the boundary layer. Panel A in Fig. 2 presents an example of such whistler mode waves detected before the Cluster spacecraft encounter the boundary layer. Although close to the Nyquist frequency at $225 \mathrm{~Hz}$, the fine-structure of the emission is evident. We believe that these waves are chorus (e.g. Burtis and Helliwell, 1976) generated either close to the equatorial plane or in magnetic field minima at higher latitudes (Tsurutani and Smith, 1977). Chorus waves propagate along the magnetic field lines and can sometimes be reflected at lower altitudes and travel back toward the region of generation (Parrot et al., 2004).

The structure of the chorus emissions should be compared with that of the whistler emissions displayed in panel B. While the chorus emission is observed on closed field lines well inside the magnetosphere, the latter whistlers are observed in the boundary layer just inside the magnetopause. The boundary layer whistlers differ from the chorus emissions in several ways. While the chorus waves appear as emissions well separated and almost regularly spaced in time, the boundary layer whistlers are more irregular. Also, chorus emissions always show time dispersion, either as risers (lowest frequencies recorded first, higher frequencies later) or fallers (the other way around). Although not very clearly seen, the strongest chorus elements in panel A are risers. The boundary layer whistlers, on the other hand, may show time dispersion (e.g., at 06:28:05 UT) but are most often non-dispersive (e.g., at 06:28:10 UT). Another outstanding feature of the boundary layer whistlers is the extremely narrow-in-time but broad-banded feature extending all the way from the lowest frequencies up to the main whistler emission above $100 \mathrm{~Hz}$. This feature, which is nicely illustrated in panel B at 06:28:10 UT, plays a key role in this paper and we will return to this later.

We conclude that two types of fine-structured whistlers in the same frequency range are present in the nearmagnetopause region. Chorus emissions are observed on field lines with magnetospheric plasma only, while the boundary layer houses whistlers showing a slightly striped pattern in the spectrogram. The remaining sections of this paper are devoted to the latter type.

\section{Wave properties and generation regions}

We begin our exploration of the boundary layer whistler mode waves by taking a closer look at the emissions associated with the magnetopause crossing at 7 February 2001 (Fig. 1). Based on the electromagnetic nature and the frequency range in which the waves are observed, we have already concluded that the observed emissions are whistler mode waves. A hodogram (not shown) confirms the expected right-handed polarization and a Poynting flux calculation (Fig. 3, panel A) shows that the whistlers propagate parallel to the background magnetic field. Panel B in Fig. 3 presents an estimate of the whistler wave phase velocity provided by the ratio $E / B$, yielding $\approx 10^{7} \mathrm{~m} / \mathrm{s}$. At $100 \mathrm{~Hz}$ this corresponds to a wavelength of $100 \mathrm{~km}$. We note that a corresponding analysis for the March 2-event is performed in Stenberg et al. (2005), where the parallel wavelength is found to be about $50-200 \mathrm{~km}$ at $100 \mathrm{~Hz}$. In that case the whistler waves instead propagates anti-parallel to the ambient magnetic field.

A feature of boundary layer whistlers, already mentioned in Sect. 4, is occasionally occurring broadbanded signatures extending to very low frequencies. One such case is evident in Fig. 2, panel B. We focus on the single stripe at 06:28:10 UT associated with a clear low frequency signature that extends (in frequency) all the way up to the whistler emission. An enlargement of this detail is shown in panel A of Fig. 4.

To understand this structure we examine the wave vector (k) direction by applying Means' method (Means, 1972). We use a magnetic field oriented coordinate system with the zaxis along $\boldsymbol{B}_{0}$. The direction of $\boldsymbol{k}$ is specified by the polar angle, $\theta$, (the angle between $\boldsymbol{k}$ and $\boldsymbol{B}_{0}$ ) and the azimuthal angle, $\phi$, (the angle in the plane perpendicular to $\boldsymbol{B}_{0}$ ). In 
Fig. 4 (panel B) the polar angle versus time and frequency is presented. For clarity all time-frequency bins with a spectral density below $8 \times 10^{-7} \mathrm{nT}^{2} / \mathrm{Hz}$ or a polar angle outside the interval $0-90^{\circ}$ are removed. The analysis does not distinguish between $\boldsymbol{k}$ and $-\boldsymbol{k}$ and we invoke the Poynting flux calculation (Fig. 3) to remove this ambiguity. We conclude that the waves propagate almost parallel to $\boldsymbol{B}_{0}$ in a $30^{\circ}$ wide cone.

In panel $\mathrm{C}$ the direction of the wave vector in the plane perpendicular to $\boldsymbol{B}_{0}$ is plotted versus time and frequency. As in the previous panel all time-frequency bins with a spectral density below $8 \times 10^{-7} \mathrm{nT}^{2} / \mathrm{Hz}$ are removed for clarity. We see a clear shift of the wave vector direction in the middle of the wave emission coinciding with the signal at lower frequencies.

The local plasma drift, $\boldsymbol{v}_{\text {drift }}$, is estimated from the ion drift (CIS/CODIF) and $\boldsymbol{E} \times \boldsymbol{B}$ (EFW and FGM). The components in the magnetic field oriented coordinate system are $v_{\text {drift }, x}= \pm 40 \mathrm{~km} / \mathrm{s}, v_{\text {drift }, y}=100-200 \mathrm{~km} / \mathrm{s}, v_{\text {drift, } z}=0$ $150 \mathrm{~km} / \mathrm{s}$. The situation is outlined in Fig. 5. It depicts a small-scale structure, in which wave generation occurs, that passes the spacecraft. The waves are assumed to propagate out from the generation region. Hence, prior to the passing of such a region the spacecraft will observe waves approaching from one direction (blue arrows), while after the region has passed the waves would reach the spacecraft from the opposite direction (yellow arrows). Thus, we interpret the observations as a wave-generating spatial structure passing the spacecraft with the plasma drift velocity. We conclude that such a generation region is recognized by a shift in the wave vector azimuthal angle, but also by a simultaneous narrow (in time) low frequency emission. During the two-minute long wave emission there are additional examples of sudden and distinct changes in the azimuthal wave vector component, all coinciding with low frequency features. Thus, the low-frequency signatures appears to be markers of whistler wave generation and we will take a closer look at them in the next section. Equivalent shifts in the azimuthal angle coinciding with low-frequency waves are found also in the March 2-event (Stenberg et al., 2005).

It should also be mentioned that in both of the discussed events the WHISPER instrument record waves in a broad frequency range around the local electron plasma frequency (4-24kHz). These high frequency waves occur at the same times as the whistler-mode waves and hence the propagation properties and/or generation/damping processes are probably related. Simultaneous whistlers and electron plasma oscillations have previously been observed, e.g., in the solar wind by Kennel et al. (1980). The WHISPER observations from the March 2-event are discussed in Canu et al. (2006).

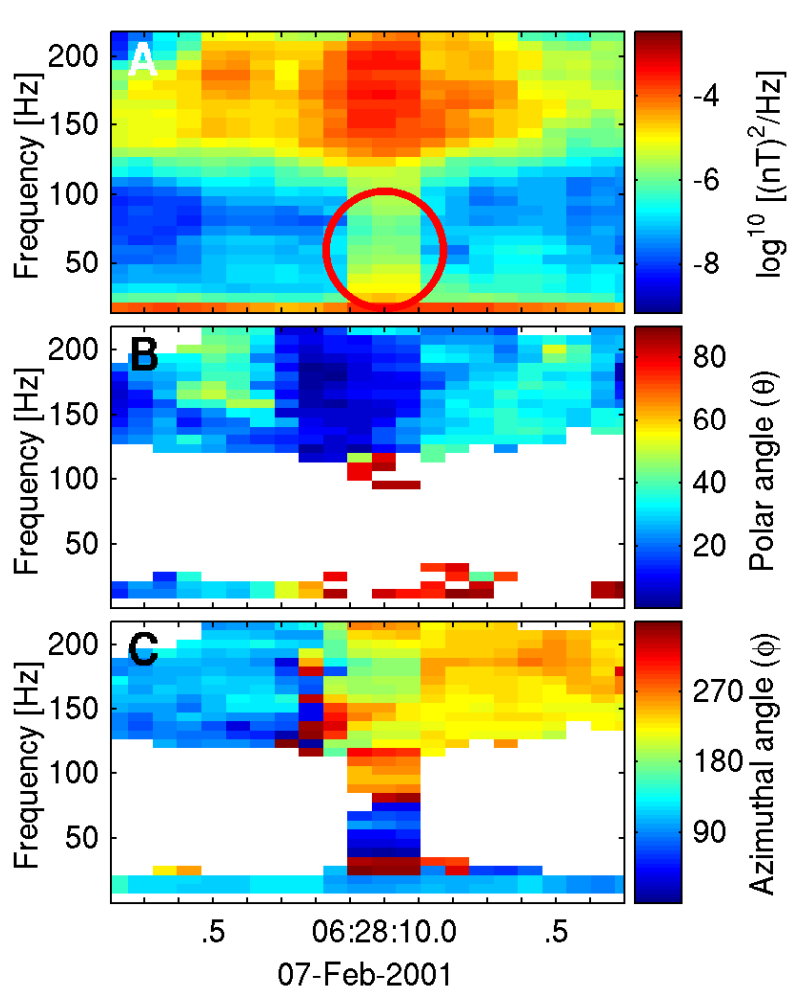

Fig. 4. Panel (A) shows a detail of the wave emission presented in Fig. 2 (panel B). We note the low frequency signature marked with a circle. Panels B and C present the results from the Means' analysis. The polar angle, $\theta$ is displayed in panel $\mathrm{B}$. The wave propagate in a $30^{\circ}$ wide cone almost parallel to $\boldsymbol{B}_{0}$. The azimuthal angle, $\phi$ is plotted versus time and frequency in the bottom panel. Coinciding with the low frequency signature, there is a shift in the azimuthal wave vector direction. We interpret this as a generation region passing the spacecraft.

\section{The low frequency signatures}

As seen in Fig. 4 the region with low frequency waves is much smaller than the region where the whistler wave emission $(>100 \mathrm{~Hz})$ is detected. In the presented spectrogram the size of the structure is just a few pixels wide and the actual width cannot be determined from there. Instead, we consider the original time series data. To easily identify the structure we low pass filter the time series, removing frequencies above $50 \mathrm{~Hz}$. Figure 6 presents the filtered time series of the three magnetic field components. It turns out that the broadbanded signature in the spectrogram is not a wave feature at all, but appears as a narrow gradient structure in the middle of the time interval shown. The amplitude of the structure is about $0.2 \mathrm{nT}$, which is same as the typical whistler-mode wave amplitude. No corresponding signature is seen in the electric wave field data (not shown). Investigating the other cases during two-minute-long emission, where a shift in the azimuthal component of the wave vector coincides with a 


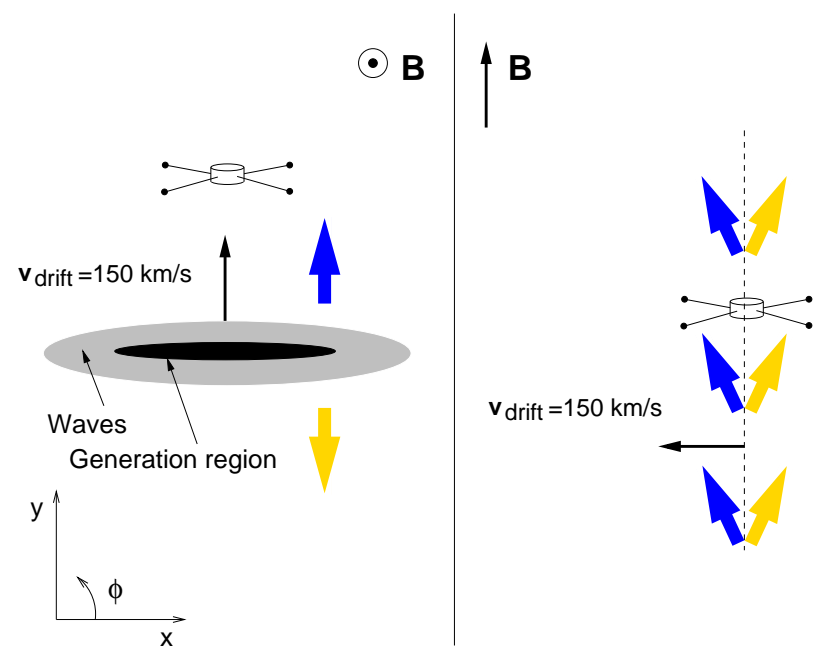

Fig. 5. A whistler wave structure containing a generation region passes the spacecraft with the plasma drift velocity. The observed wave vector directions are illustrated by colored arrows, where the colors are the same as used in panel $\mathrm{C}$ in Fig. 4. The waves are assumed to propagate out of the generation region, with an angle between $\boldsymbol{k}$ and $\boldsymbol{B}_{0}$ in the range $0-30^{\circ}$. As the generation region pass we record a sudden shift in the propagation direction in the plane perpendicular to $\boldsymbol{B}_{0}$. Note that we only indicate the perpendicular component of the plasma drift velocity.

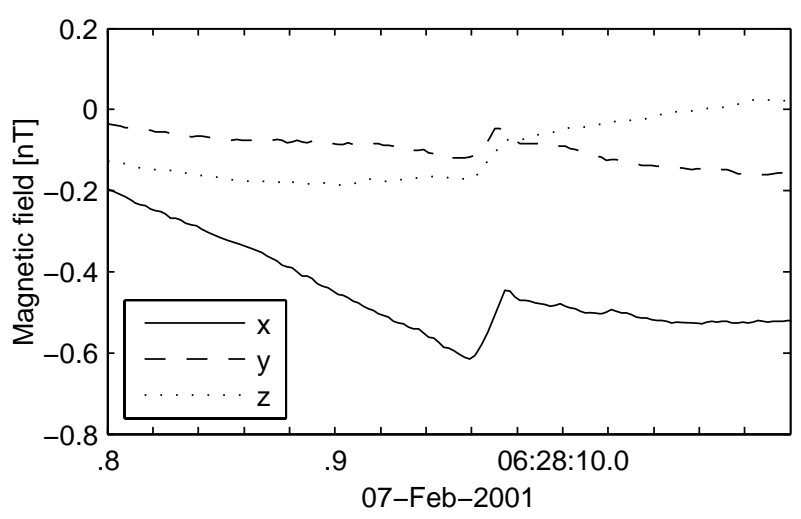

Fig. 6. The time series from STAFF-SC on Cluster 2 is low pass filtered with the maximum frequency $50 \mathrm{~Hz}$. The low frequency emission seen in the spectrogram (e.g. Fig. 4) now appear as gradient structures in the components of the magnetic field at 06:28:09.96 UT.

low-frequency signature, we find similar features: The time series data reveals not low frequency waves but instead magnetic field structures. Unfortunately, we cannot find a case where we are convinced that the same singular structure is seen by two different spacecraft. Hence, we have no means of determining the temporal and spatial characteristics of the structures. Fortunately, the second event we consider turns out to be a source of further knowledge.

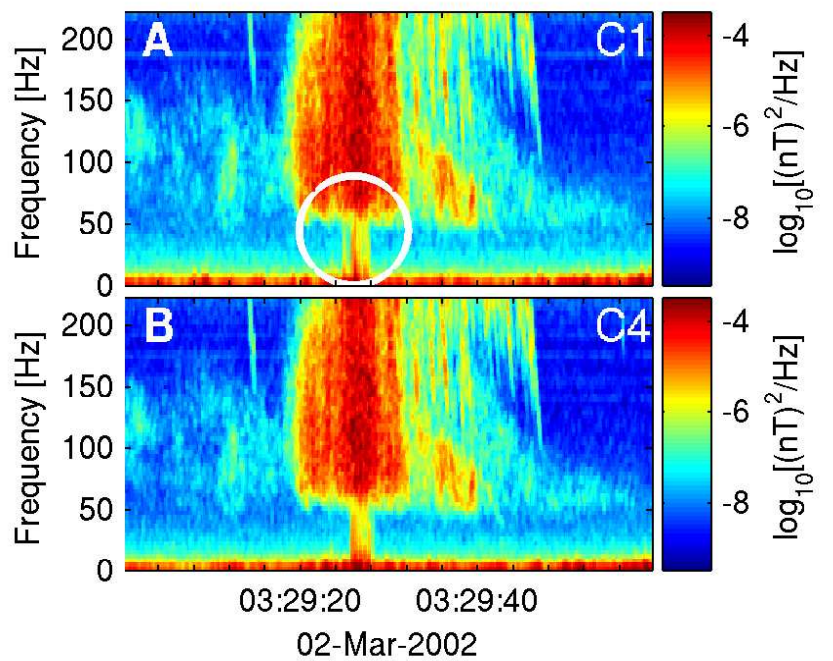

Fig. 7. Time-frequency spectrograms for spacecraft $\mathrm{C} 1$ and $\mathrm{C} 4$ observed in the boundary layer about $1.5 \mathrm{~min}$ before an outbound magnetopause crossing. The magnetic wave field is recorded by STAFF-SC. The waves with frequencies $>50 \mathrm{~Hz}$ are whistler mode waves. In the middle of the two panels a low frequency signature is seen (indicated by a circle in panel $\mathbf{A}$ ).

Figure 7 shows frequency-time spectrograms of the magnetic field observed by Cluster 1 (C1) and Cluster 4 (C4) on 2 March 2002, prior to an outbound magnetopause crossing at 03:31 UT. All data is recorded in a boundary layer, where both magnetospheric and magnetosheath plasma are present. We note the wave activity at whistler mode wave frequencies $(>50 \mathrm{~Hz})$ but also a low-frequency signature (marked by a circle in panel A), that seems to be seen by both spacecraft. As in the previously shown cases this signature coincides with a clear shift in the azimuthal wave vector component (Stenberg et al., 2005).

Low-pass filtering the time series yields the result presented in Fig. 8. The three magnetic wave field components are plotted versus time for the two spacecraft $\mathrm{C} 1$ and $\mathrm{C} 4$. The coordinate system used is background magnetic field oriented with the $z$-component along the ambient field. We see that there are several different magnetic structures present during the time interval shown. They are seen in all three field components and they all have about the same amplitude. In particular, we note that the parallel $(z)$ component is no different from the perpendicular ( $x$ and $y$ ) components. Again, the amplitude of the structures is comparable to the whistler wave amplitude.

Although it is obvious that the same signatures truly are captured by both satellites, their appearance differ considerably between the spacecraft. Furthermore, there is a small time shift between the recordings, where $\mathrm{C} 4$ lags $\mathrm{C} 1$ with $0.01 \mathrm{~s}$. It is worth mentioning that the sequence containing this type of magnetic structures is actually much longer. It starts at 03:29:24.7 UT and lasts for $3 \mathrm{~s}$. The magnetic 


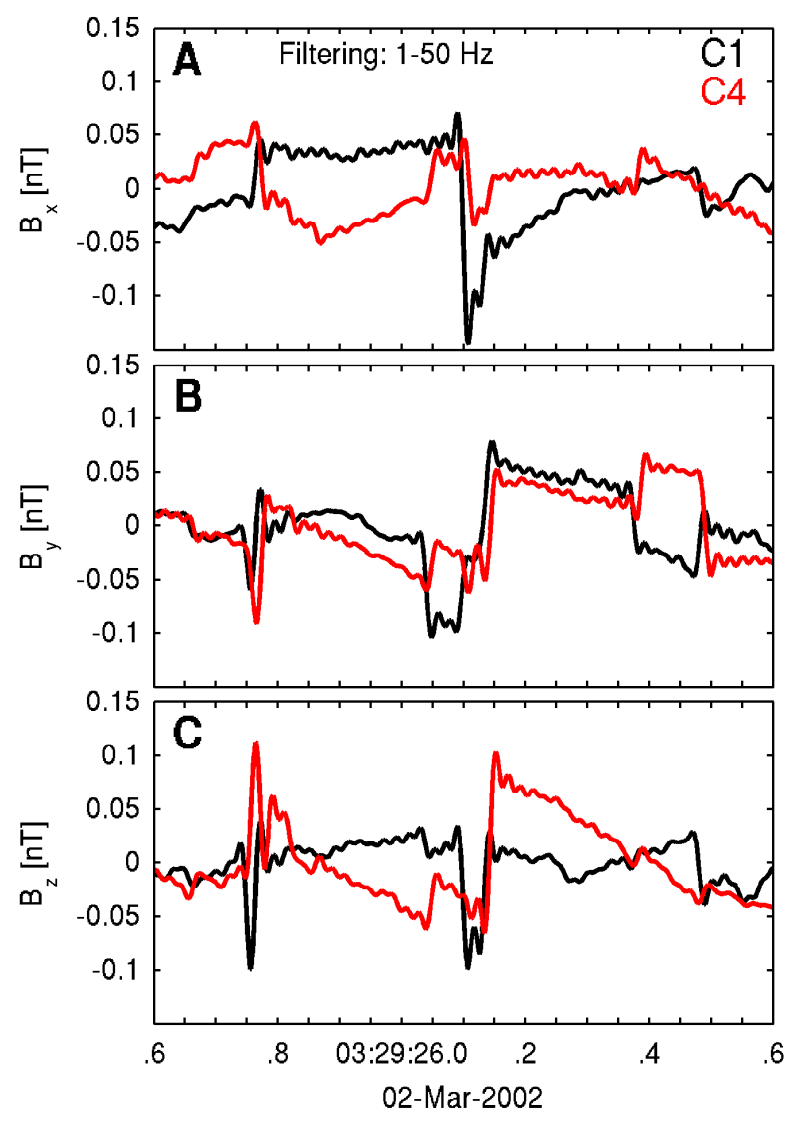

Fig. 8. Time series of the three magnetic wave field components (STAFF-SC) observed by $\mathrm{C} 1$ (black) and C4 (red). The data are band pass filtered between $1-50 \mathrm{~Hz}$. The coordinated system used is magnetic field oriented with the $z$-component along the ambient field.

structures shown in Fig. 8 are typical and the time span is chosen to make the time shift between the spacecraft clearly visible.

To sort out the situation we notice that the two spacecraft are almost on the same magnetic field line, separated by $100 \mathrm{~km}$ along the field. Hence, for a signal to reach C4 0.01 seconds after being detected on $\mathrm{C} 1$ it must move with the speed $10^{7} \mathrm{~m} / \mathrm{s}$ anti-parallel to the field. This velocity is the group velocity of the whistler waves. We can then estimate the parallel size of magnetic step-like structures in Fig. 8. Assuming a typical jump takes about $0.01 \mathrm{~s}$ the corresponding size is $100 \mathrm{~km}$, which is about an ion inertial length $\left(c / \omega_{p i}\right)$.

The perpendicular size is likely to be of the order of the spacecraft separation, noting that the observations made by $\mathrm{C} 1$ and $\mathrm{C} 4$ are considerably different. The satellite separation perpendicular to the background magnetic field is $30 \mathrm{~km}$.

We argue that the discovered magnetic step-like features are current structures moving together with the whistler waves at the group velocity and with a perpendicular size of

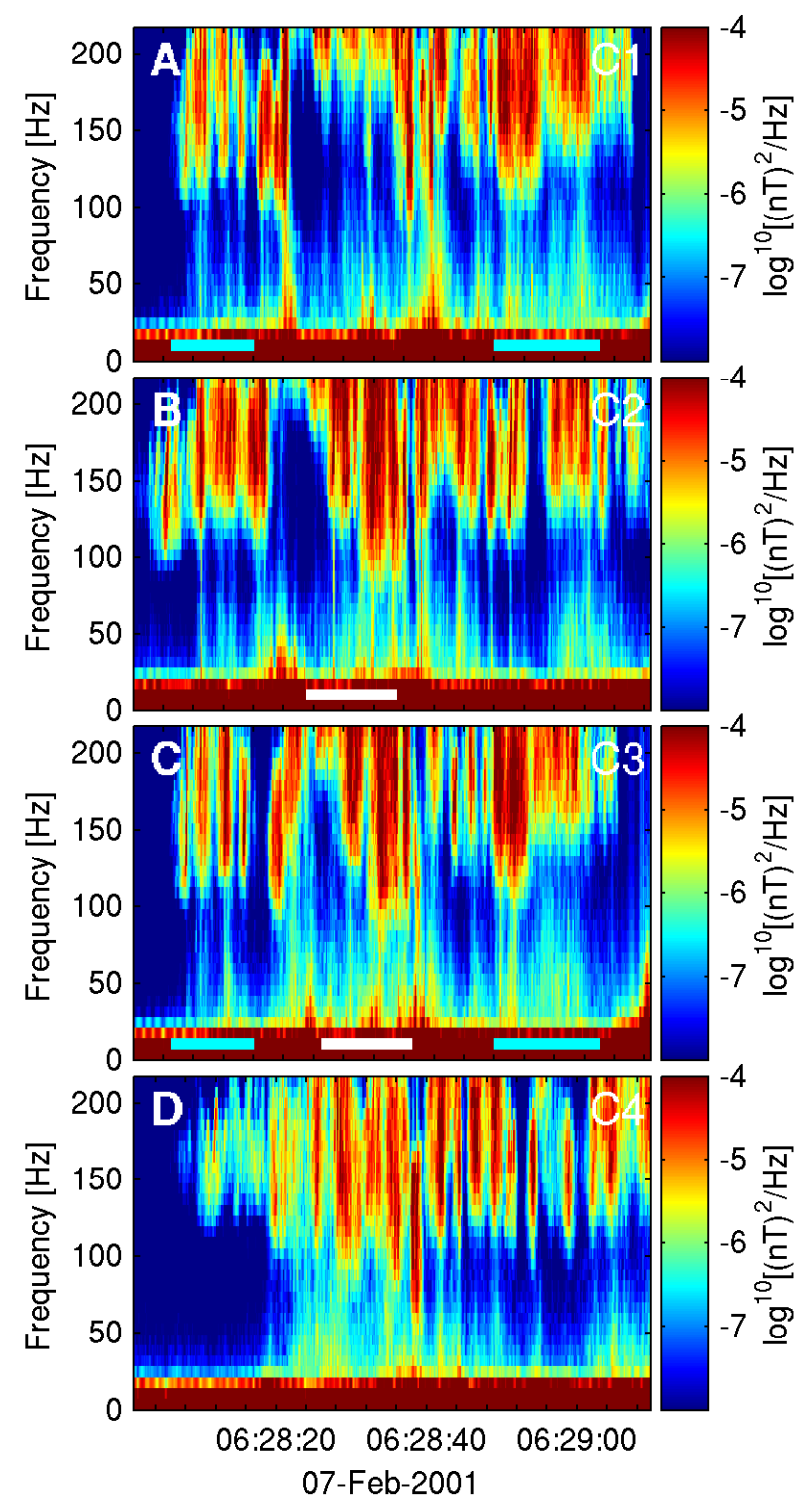

Fig. 9. Time-frequency spectrograms (STAFF-SC) for the four spacecraft. The horizontal lines mark regions where two satellites appear to record the same emissions.

$30-100 \mathrm{~km}$ (less than an ion inertial length). These features are commonly observed within or close to the regions where the whistler mode waves appear to be generated.

\section{$7 \quad$ Size matters}

Returning to an overview of the whistler wave emission recorded on 7 February 2001 (Fig. 9), we proceed with estimating the three-dimensional size of the singular stripe-like whistler emissions. Figure 9 shows about $70 \mathrm{~s}$ of data. All four Cluster spacecraft observe whistler waves while crossing the boundary layer. A comparison between the emissions 


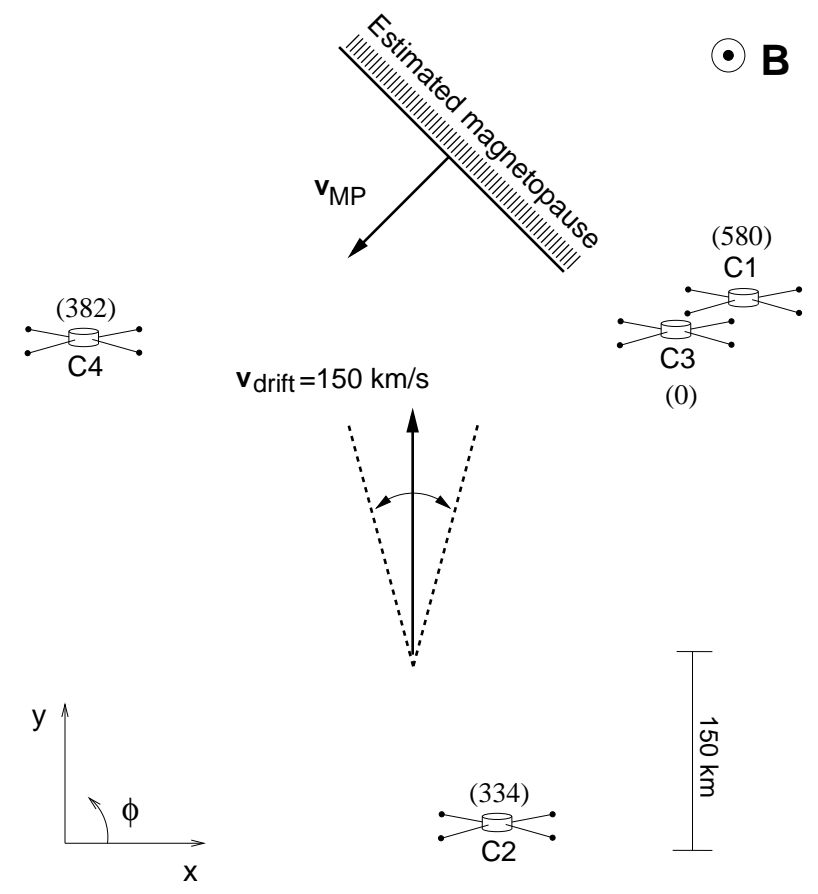

Fig. 10. The location of the Cluster spacecraft in the magnetic field reference frame. The background magnetic field is directed out of the figure. An estimate of the magnetopause normal is shown. Illustrated is also the plasma drift, which is varying during the time interval analyzed. The numbers in parenthesis are the spacecraft distances (in $\mathrm{km}$ ) in the direction parallel to $\boldsymbol{B}_{0}$ with respect to the reference Cluster 3.

seen by the different satellites allows us to add new pieces of information.

We realize that although all panels show the similar striped wave emission character, it is not easy to identify the same structures on the different spacecraft. We concentrate on a few time intervals, where such an identification is possible.

Comparing Cluster 2 (C2) and Cluster 3 (C3) we find a sequence in the middle of the time interval shown, where the spacecraft appear to capture the same wave emissions. The roughly $12 \mathrm{~s}$ long sequence is indicated by a horizontal white line in the two panels. There is a timeshift of about two seconds between the observations, where $\mathrm{C} 2$ detects the emissions before $\mathrm{C} 3$ does. This time delay fits nicely with the estimates of the plasma drift velocity and the location of the spacecraft.

Figure 10 presents the situation in the magnetic field oriented system used throughout the paper. The location of the four spacecraft is indicated as well as an estimate of the magnetopause normal. Note though that all spacecraft are well on the magnetospheric side of the boundary. The plasma drift is varying as indicated in Fig. 10 but is mainly in the $\mathrm{y}$-direction. With a perpendicular distance of $400 \mathrm{~km}$ between $\mathrm{C} 2$ and $\mathrm{C} 3$ and an averaged plasma drift during the time period of $150 \mathrm{~km} / \mathrm{s}$, we would expect a time difference of a few seconds between the observations if the structures are fixed with respect to the plasma. In addition, each individual stripe seems to be about one second long in the timefrequency spectrograms. That means their physical size is about $150 \mathrm{~km}$. The typical width of a stripe can be estimated from Figs. 2 and 4. Hence, we conclude that the whistler wave emissions are $150 \mathrm{~km}$ wide, spatial regions moving with the background plasma. Furthermore, we conclude that the structures are preserved for at least a couple of seconds.

It should also be noted that neither $\mathrm{C} 1$ nor $\mathrm{C} 4$ observe these whistler wave structures. Thus, these wave regions move with the plasma from $\mathrm{C} 2$ to $\mathrm{C} 3$ without hitting the other two satellites. Hence, the dimension perpendicular to the drift velocity in the plane shown in Fig. 10 must be smaller than the spacecraft separation $(\approx 600 \mathrm{~km})$.

Next, we compare spacecraft $\mathrm{C} 1$ and $\mathrm{C} 3$. As seen from Fig. 10, these two satellites are close to each other in the plane perpendicular to $\boldsymbol{B}_{0}$. $\mathrm{C} 1$ and $\mathrm{C} 3$ detect the same emissions on two occasions, one at the beginning and one towards the end of the time interval shown in Fig. 9. These regions are marked with horizontal light blue lines in panels $\mathrm{A}$ and $\mathrm{C}$. It is difficult to observe any time shift at all between the observations in either of the cases. This is not surprising considering the short distance between the spacecraft in the direction perpendicular to $\boldsymbol{B}_{0}$. With a plasma drift of $150 \mathrm{~km} / \mathrm{s}$ the time difference would never exceed fractions of a second. Along $\boldsymbol{B}_{0}$ the waves themselves propagate at a speed of $10000 \mathrm{~km} / \mathrm{s}$ and two satellites on the same field line separated by no more than $600 \mathrm{~km}$ will record the waves almost simultaneously. The observation confirms our conclusions from comparing $\mathrm{C} 2$ and $\mathrm{C} 3$.

We emphasize that we determine the width of the whistler emission structure in the direction parallel to $\boldsymbol{v}_{\text {drift }}$, but we have no means to directly measure the size perpendicular to the drift velocity. From the $\mathrm{C} 2 / \mathrm{C} 3$ comparison can only say that it is less than $600 \mathrm{~km}$. However, we also note that all the striped whistler wave emissions seem to have about the same size in the spectrogram. This implies that the structures are either almost symmetric in the plane perpendicular to $\boldsymbol{B}_{0}$, or they are oriented so that the minor/major axis is always parallel to the drift velocity. While some kind of ordering with respect to $\boldsymbol{v}_{\text {drift }}$ is not impossible, we believe that the effect is mostly due to the fact that the structures are comparable in size in both directions perpendicular to $\boldsymbol{B}_{0}$. Figure 5 represents a compromise with one perpendicular dimension slightly larger and aligned perpendicular to the drift velocity.

The generation of the whistler mode waves probably takes place in smaller regions in the middle of the observed emissions, where also the current structures discussed in the previous section are found.

In summary, we believe that the regions in which the whistler waves are observed, as well as the presumably smaller generation regions within them, are tube-like structures. The regions, where waves are observed have a 

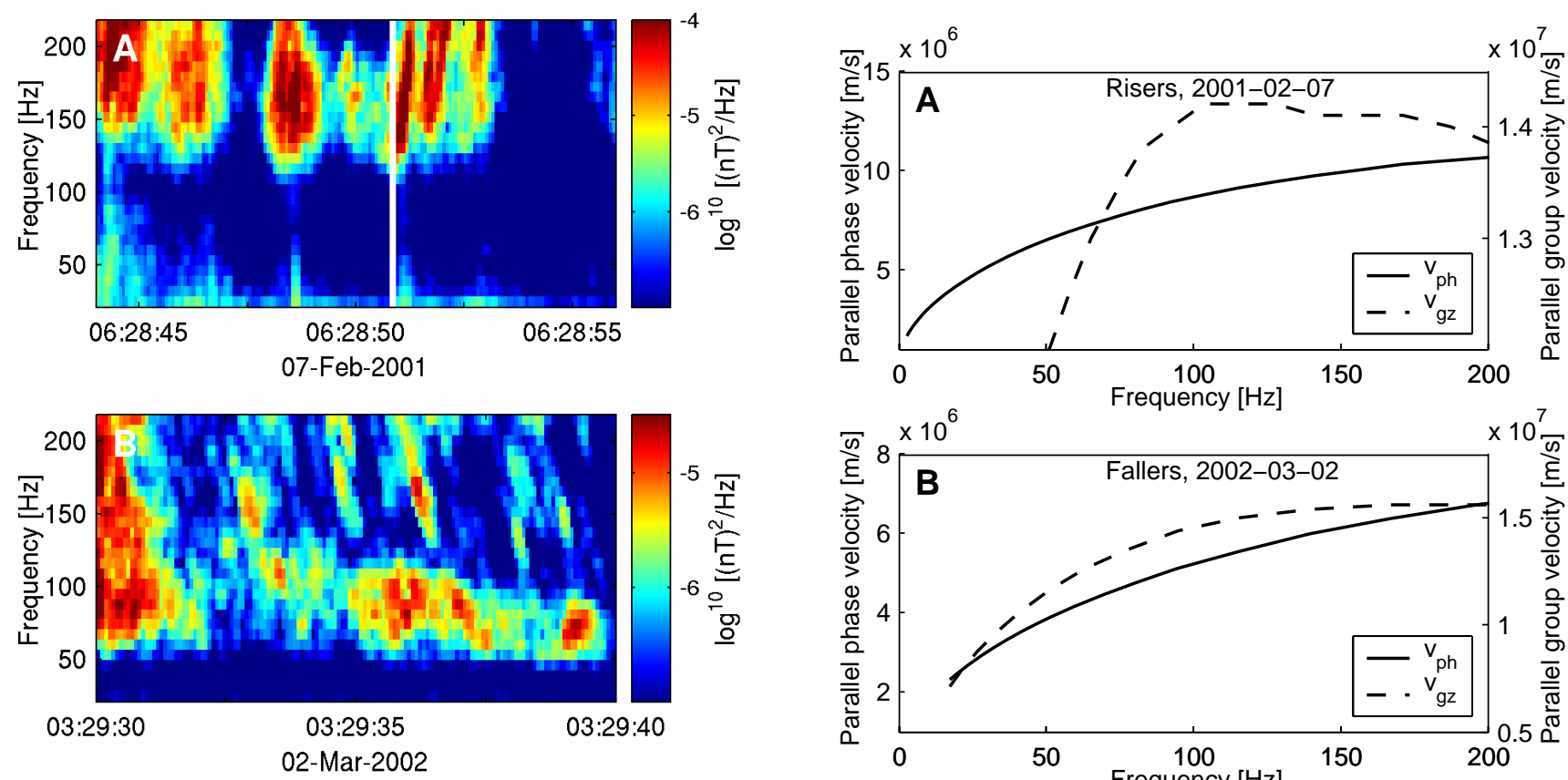

Fig. 11. Examples of risers and fallers. Panel (A) is an example of risers found by Cluster 2 in the boundary layer on 7 February 2001. The example in panel (B) is taken from a magnetopause crossing on 2 March 2002. The presented whistler waves are observed by Cluster 1 in a boundary layer with the same mix of high and low energy electrons. Several fallers are clearly visible. In both panels data from STAFF-SC is used.

perpendicular size parallel to the drift velocity of about 100 $200 \mathrm{~km}$. The size perpendicular to drift velocity is most probably comparable to, but definitely less than, $600 \mathrm{~km}$.

\section{The third dimension: risers and fallers}

The extension of the whistler mode wave stripes along the field lines still has to be estimated. This section will be devoted to that issue, and we will focus on the dispersive elements observed in the wave emission.

The accepted view of chorus (cf. Sect. 4) is that the rising and/or falling tones are created by the particular mechanism generating the waves (Helliwell, 1967). This is in contrast to lightning-generated whistler waves in the ionosphere, where the observed time dispersion is due to a propagation effect, (e.g., Storey, 1953). Boundary layer whistlers are observed not far from regions of chorus and they sometimes show a clear time dispersion. This is shown already in Fig. 2. Another example of risers taken from the same event is displayed in Fig. 11 (panel A). Whistlers in the boundary layer may also appear as fallers. Panel B in Fig. 11 shows such a case taken from the magnetopause crossing on 2 March 2002. It is not clear what causes the time dispersion of boundary layer whistlers, propagation effects or a chorus-like gener-

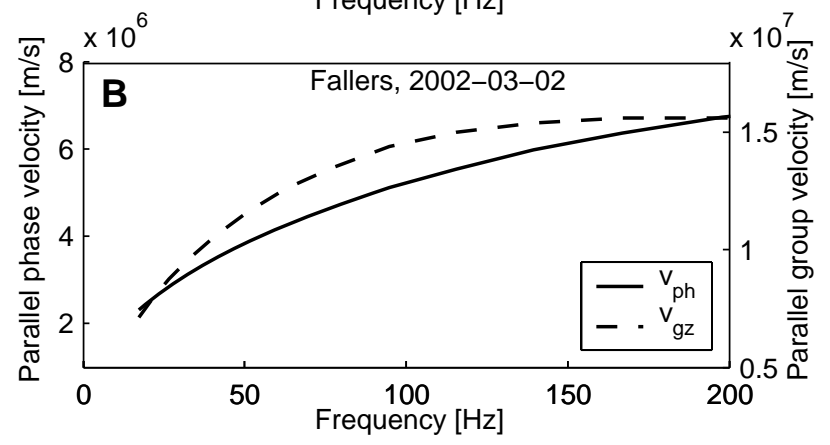

Fig. 12. The dashed lines show the parallel group velocity, $v_{g z}$, versus frequency for two cases. Panel (A) presents a case where risers can be formed above $100 \mathrm{~Hz}$. In panel (B) the group velocity increases with frequency in the interval $0-200 \mathrm{~Hz}$, which would give rise to fallers. The phase velocity, $v_{p h}$, (solid lines) increases with frequency for both cases.

ation mechanism. However, the boundary layer whistlers observed on 7 February 2001 show a large number of sudden changes of the azimuthal component of the wave vector indicating that the satellites pass through several generation regions. In all these cases the whistler wave elements are non-dispersive. This speaks in favor of explaining the time dispersion of boundary layer whistlers as a propagation effect.

To investigate if it is possible that the risers and fallers observed in the boundary layer are due to propagation effects we investigate the wave properties using the dispersion solver WHAMP (Rönnmark, 1982). The plasmas observed in the two events shown in Fig. 11 are modelled as presented in Table 1. For the risers' event we use a plasma density of $0.7 \mathrm{~cm}^{-3}$ and a background magnetic field of $20 \mathrm{nT}$. The ions are isotropic $5 \mathrm{keV}$ protons, the magnetosheath electrons are regarded isotropic with a thermal energy of $70 \mathrm{eV}$, and the hot magnetospheric electrons are modelled using a $1 \mathrm{keV}$ losscone distribution. The model describing the fallers event is discussed in Stenberg et al. (2005). In that case the total density is higher and there is no loss cone distribution. Instead, the magnetosheath electrons have a temperature anisotropy.

Figure 12 shows that both risers and fallers are possible to explain using these plasma models. Panel A shows the attempt to model the situation in the major event discussed 
Table 1. The plasma models used to recreate risers and fallers. All components are assumed to be Maxwellians, in one case including a loss-cone, as indicated in the table. The models are based on PEACE and CIS observations. The model for the 2 March 2002 event is previously used in Stenberg et al. (2005).

\begin{tabular}{|c|c|c|c|}
\hline \multicolumn{4}{|c|}{ FEBRUARY 7, 2001: RISERS } \\
\hline Species & Density $\left[\mathrm{cm}^{-3}\right]$ & $T_{\|}[\mathrm{eV}]$ & $T_{\perp}[\mathrm{eV}]$ \\
\hline $\mathrm{H}^{+}$ & 0.7 & 5000 & 5000 \\
\hline $\mathrm{e}^{-}$ & 0.6 & 70 & 70 \\
\hline $\mathrm{e}^{-}$ & 0.1 & 1000 & 1000 (Loss cone) \\
\hline \multicolumn{4}{|c|}{$\boldsymbol{B}_{0}=20 \mathrm{nT}$} \\
\hline \multicolumn{4}{|c|}{ MARCH 2, 2002: FALLERS } \\
\hline Species & Density $\left[\mathrm{cm}^{-3}\right]$ & $T_{\|}[\mathrm{eV}]$ & $T_{\perp}[\mathrm{eV}]$ \\
\hline $\mathrm{H}^{+}$ & 3.0 & 2000 & 2000 \\
\hline $\mathrm{e}^{-}$ & 2.6 & 40 & 10 \\
\hline $\mathrm{e}^{-}$ & 0.4 & 400 & 400 \\
\hline \multicolumn{4}{|c|}{$\boldsymbol{B}_{0}=30 \mathrm{nT}$} \\
\hline
\end{tabular}

in this paper (7 February 2001). Here the group velocity decreases with frequency above $100 \mathrm{~Hz}$, producing risers in this frequency range. Lower frequencies propagate faster and arrive a head of the higher frequencies. In panel B both the group and phase velocities increase with frequency in the interval $0-200 \mathrm{~Hz}$. Parallel propagating whistlers would in such a plasma evolve into fallers as the high frequencies travel faster.

More work is required to fully understand the dependence of the wave properties on different parameters in the models. Our aim is merely to show that both risers and fallers are possible outcomes of realistic plasma models. The brief investigation show that the behavior of the group velocity is sensitive to the relative temperatures of the two electron components as well as to the width and depth of any loss-cone.

Viewed as a propagation effect, the observed time dispersion is consistent with a picture where the whistler emissions extend great distances along the magnetopause. For instance, consider the riser in panel A of Fig. 11, where the time difference between waves at 100 and $200 \mathrm{~Hz}$ is about a quarter of a second. Furthermore, assume that the group velocities at $100 \mathrm{~Hz}\left(v_{100}\right)$ and $200 \mathrm{~Hz}\left(v_{200}\right)$ are given by the the analytical results presented in Fig. 12. Then, to produce the observed time difference the wave must travel a distance, $s$, given by $s / v_{200}-s / v_{100}=0.25 \mathrm{~s}$. With $v_{200}=1.38 \times 10^{7} \mathrm{~m} / \mathrm{s}$ and $v_{100}=1.44 \times 10^{7} \mathrm{~m} / \mathrm{s}, s=12 \mathrm{R}_{\mathrm{E}}$. The numerical result should not be regarded an exact value, but serve as an indication of that the whistler wave emission regions may extend for long distances along the field lines.

\section{The global picture}

The whistler mode waves occur on field lines with a mixture of magnetospheric and magnetosheath plasma. Hence, these are, or have recently been, open field lines in order to allow inflow of magnetosheath plasma. Moreover, Stenberg et al. (2005) suggests that the generation of the waves is due to an electron anisotropy, resulting from connecting magnetospheric and magnetosheath field through the magnetopause, i.e., magnetic reconnection. This result agrees also with Vaivads et al. (2007). However, for both the events considered in this paper a northward interplanetary magnetic field (IMF) is observed. We would therefore expect the major reconnection X-line to be located tailward of the cusp, where the IMF and the magnetospheric field lines are antiparallel. The Cluster spacecraft cross the magnetopause at high latitude sunward of the cusp and should not record any signs of this process. Neither do we observe any clear rotation of the magnetic field in the magnetopause plane, when the Cluster spacecraft pass this boundary minutes after the wave emission encounters. Hence, we can not provide any strong evidence of reconnection southward of the cusp. If such active processes exist at this time, our results suggests they are probably local, patchy and/or intermittent.

\section{Discussion}

In a previous paper (Stenberg et al., 2005) we suggest that the whistler waves just inside the magnetopause are generated in thin sheets. Now we are inclined to believe that whistler waves are generated and propagate in tube-like, perhaps even cylindrically symmetric, structures. The generation is located in the center of the tubes. It is not the first time tubelike structures have been found in this region of space. Examples of such spatial structures are discussed in, e.g., Rezeau et al. (1993), Alexandrova et al. (2004) and Alexandrova et al. (2006), who study low-frequency Alfvénic activity at the magnetopause and in the magnetosheath respectively.

This study presents evidence of a large amount of tightly packed wave generating tubes in the magnetopause boundary layer. Moreover, the apparent mix of locally generated waves and waves that have propagated substantial distances suggests that a large region of the boundary layer is characterized by such small-scale structures. In Stenberg et al. (2005) it is argued that a whistler generation region provide a direct link to the reconnection diffusion region. The magnetospheric electrons are found to disappear at the same time as the whistlers are observed. From this it is argued that the whistlers are observed on field lines directly connected to a reconnection region, thereby allowing the magnetospheric electrons to escape. The duration of a single generation region in the present paper is too short to allow us to study the electrons in such detail. However, the event is in other respects very similar to that studied in Stenberg et al. 
(2005). Hence, a speculation is that also in the present case the whistlers are observed on field lines directly connected to diffusion regions at the magnetopause. In that case, the tube-like emission structures found indicate a patchy and/or intermittent reconnection geometry. We note, however, that there are no global signs of reconnection processes sunward of the cusp. Bursty whistler-mode waves observed by the Polar spacecraft in the outer cusp have also been suggested to originate from a diffusion region (Pickett, et al., 2001).

Another interesting question is the small perpendicular size of the whistler wave elements. Observations as well as simulations show that magnetic field and density perturbations often correlate with whistler mode activity, (Moullard, 2002; Eliasson and Shukla, 2005a). Enhancements or cavities duct whistler waves along the ambient magnetic field. In our case we do not observe any field or density fluctuations correlating with the individual wave elements but the situation, especially for the February 7-event, is complicated with overlapping wave regions. Thus, we cannot rule out this explanation.

We also lack the full explanation of the step-like magnetic field structures observed within the waves. They are observed in or close to regions where the waves are generated and where the waves are most intense, although the intensity is not extraordinary. It is known that whistler mode waves can be subject to linear or non-linear self-focusing, (Eliasson and Shukla, 2005b). One suggestion is then that such a process is responsible for the observed phenomena.

\section{Summary of conclusions}

The focus of this paper is whistler mode waves observed close to the magnetopause in the boundary layer on the magnetospheric side of the boundary. In a time-frequency spectrogram the waves appear as stripes, narrow in time and broader in frequency. With the help of the four-spacecraft mission Cluster we are able to determine both the threedimensional size of the wave emissions and their internal structure. The main conclusions are summarized below:

- The whistler mode waves are observed in tube-like structures in space, extending a long way along the field lines (see below) but limited to about an ion inertial length (or less) in the perpendicular direction.

- Some of the whistler wave elements are non-dispersive. An investigation of the wave vector direction reveals a sudden and distinct change of the azimuthal component in the middle of such emission elements. We argue that these are regions, where waves are generated. As a consequence, plasma of the boundary layer is organized in tubes, which favor whistler wave generation.

- Within the presumed locally generated whistler waves we detect ion-scale current structures, that propagate with the whistler wave group velocity in the same direction as the waves. The amplitude of the current structures is comparable to the whistler wave amplitude.

- Other whistler emission elements are clearly dispersive. They manifest themselves as risers or as fallers. Using simple plasma models we conclude that it is possible to explain both cases as a propagation effect. This allows us to estimate the extension of the emissions along the ambient field. We find that the whistler waves propagate at least $10 \mathrm{R}_{\mathrm{E}}$.

- The large amount of detected generation regions (identified as non-dispersive emission elements) indicates a high density of the tube-like structures in the plasma. Referring to Stenberg et al. (2005), where a whistler generation region is believed to couple directly to the reconnection site, we speculate that the observed pattern of tube-like regions reflects ongoing intermittent and/or patchy reconnection.

Acknowledgements. We are grateful to A. Balogh and S. Buchert for supplying FGM data to this study and for useful discussions.

Topical Editor F. D'Andrea thanks two anonymous referees for their help in evaluating this paper.

\section{References}

André, M., Vaivads, A., Buchert, S. C., et al.: Thin electron-scale layers at the magnetopause, Geophys. Res. Lett., 31, L03803, doi:10.1029/2003GL018137, 2004.

Alexandrova, O., Mangeney, A., Maksimovic, M., et al.: Cluster observations of finite amplitude Alfvén waves and small-scale magnetic filaments downstream of a qusi-perpendicular shock, J. Geophys. Res., 109, A05207, doi:10.1029/2003JA010056, 2004.

Alexandrova, O., Mangeney, A., Maksimovic, M., et al.: Alfvén vortex filaments observed in magnetosheath downstream of a quasi-perpendicular bow shock, J. Geophys. Res.,111, A10, 10.1029/2006JA011934, 2006.

Balogh, A., Dunlop, M. W., Cowley, S. W. H., et al.: The Cluster Magnetic Field Investigation, Space Sci. Rev., 79, 65-91, 1997.

Burtis, W. J. and Helliwell, R. A.: Magnetospheric chorus: Occurrence patterns and normalized frequency, Planet. Space Sci., 24, 1007-1024, 1976.

Canu, P., Décréau, P., Escoffier, S., et al.: A search for electron scale structures close to the magnetopause, Cluster and Double Star Symposium, 5th Anniversity of Cluster in Space, ESA SP598, European Space Agency, 2006.

Chapman S., and Ferraro, V. C. A.: A new theory of magnetic storms, Terrestr. Magn. Atmos. Elec., 36, 77-97, 171-186, 1931.

Cornilleau-Wehrlin, N., Chauveau, P., Louis, S., et al.: The Cluster spatio-temporal analysis of field fluctuations (STAFF) experiment, Space Sci. Rev., 79, 107-136, 1997.

Cornilleau-Wehrlin, N., Chanteur, G., Perraut, S., et al.: First results obtained by the Cluster STAFF experiment, Ann. Geophys., 21, 437-456, 2003,

http://www.ann-geophys.net/21/437/2003/. 
Décréau, P. M. E., Fergeau, P., Krannosels'kikh, M., et al.: WHISPER, a resonance sounder and wave analyser: Performance and perspectives for the Cluster mission, Space Sci. Rev., 79, 157193, 1997.

Dungey, J. W.: Interplanetary magnetic fields and the auroral zones, Phys. Rev. Lett., 6, 47-48, 1961.

Eastman, T. E., Hones, Jr., E., W., Bame, S. J., et al.: The magnetospheric boundary layer: Site of plasma, momentum, and energy transfer from the magnetosheath into the magnetosphere, Geophys. Res. Lett., 3, 685-688, 1976.

Eliasson, B. and Shukla, P. K.: Three-dimensional dynamics of nonlinear whistlers in plasmas, Phys. Lett. A, 348, 51-57, 2005a.

Eliasson, B. and Shukla, P. K.: Linear self-focusing of whistlers in plasmas, New Journal of Physics, 7(95), 1-10, 2005 b.

Escoubet, C. P., Schmidt, R., and Goldstein, M.: Cluster - Science and Mission Overview, Space Sci. Rev., 79, 11-32, 1997.

Escoubet, C. P., Fehringer, M., and Goldstein, M.: The Cluster Mission, Ann. Geophys., 19, 1197-1200, 2001, http://www.ann-geophys.net/19/1197/2001/.

Gurnett, D. A., Anderson, R. R., Tsurutani, B. T., et al.: Plasma Wave Turbulence at the Magnetopause: Observations From ISEE 1 and 2, J. Geophys. Res., 84, 7043-7058, 1979.

Gustafsson, G., Boström, R., Holback, B., et al.: The electric field and wave experiment for the Cluster mission, Space Sci. Rev., 79, 137-156, 1997.

Haerendel, G., Paschmann, G., Sckopke, N., et al.: The frontside boundary layer of the magnetosphere and the problem of reconnection, J. Geophys. Res., 83, 3195-3216, 1978.

Helliwell, R. A.: A Theory of Discrete VLF Emissions from the Magnetosphere, J. Geophys. Res., 72, 4773-4790, 1967.

LaBelle, J. and Treumann, R. A.: Plasma waves at the dayside magnetopause, Space Sci. Rev., 47, 175-202, 1988.

Johnstone, A. D., Alsop, C., Burge, S., et al.: PEACE: A plasma electron and current experiment, Space Sci. Rev., 79, 351-398, 1997.

Kennel, C. F., Scarf, F. L., Coroniti, F. V., et al.: Correlated Whistler and Electron Plasma Oscillation Bursts Detected on ISEE-3, Geophys. Res. Lett., 7, 129-132, 1980.

Maksimovic, M., Harvey, C. C., Santolík, O., et al.: Polarization and propagation of lion roars in the dusk side magnetosheath, Ann. Geophys., 19, 1429-1438, 2001, http://www.ann-geophys.net/19/1429/2001/.

Means, J. D.: Use of the Three-Dimensional Covariance Matrix in Analyzing the Polarization Properties of Plane Waves, J. Geophys. Res., 77, 5551-5559, 1972.

Moullard, O., Masson, A., Laakso, H., et al.: Density modulated whistler mode emissions observed near the plasmapause, Geophys. Res. Lett., 29(20), 1975, doi:10.1029/2002GL015101, 2002.

Mozer, F. S.: Criteria for and statistics of electron diffusion regions associated with subsolar magnetic field reconnection, J. Geophys. Res., 110, A12222, doi:10.1029/2005JA011258, 2005.

Olson J. V, Hozer, R. E., and Smith, E. J.: High-Frequency Magnetic Fluctuations Associated with the Earth's Bow Shock, J. Geophys. Res., 74, 4601-4617, 1969.

Onofri, M., Primavera, L., Malara, F., and Veltri P.: Threedimensional simulations of magnetic reconnection in slab geometry, Phys. Plasmas, 11, 4837-4846, 2004.

Parrot, M., Santolík, O., Gurnett, D. A., et al.: Characteristics of magnetospherically reflected chorus waves observed by CLUSTER, Ann. Geophys., 22, 2597-2606, 2004, http://www.ann-geophys.net/22/2597/2004/.

Paschmann, G.: Plasma acceleration at the Earth's magnetopause: Evidence for reconnection, Nature, 282, 243-246, 1979.

Pedersen, A., Décréau, P., Escoubet, C.-P., et al.: Four-point high time resolution information on electron densities by the electric field experiment (EFW) on Cluster., Ann. Geophys., 19, 14831489, 2001, http://www.ann-geophys.net/19/1483/2001/.

Pickett, J. S., Menietti, J. D., Dowell, J. H., et al.: Polar spacecraft observations of the turbulant outer cusp/magnetopause boundary layer of Earth, Nonlinear Proc. Geophys., 6, 195-204, 1999.

Pickett, J. S., Franz, J. R., Scudder, J. D., et al.: Plasma waves observed in the cusp turbulent boundary layer: An analysis of high time resolution wave and particle measurements from the Polar spacecraft, J. Geophys. Res., 106, 19081-19099, 2001.

Rème, H., Bosqued, J. A., Sauvaud, J. A., et al.: The Cluster Ion Spectrometry (CIS) Experiment, Space Sci. Rev., 79, 303-350, 1997.

Retinò, A., Vaivads, A., André, M., et al.: The structure of the separatrix region close to a magnetic reconnection $\mathrm{X}$ line: Cluster observations, Geophys. Res. Lett., 33, L06101, doi:10.1029/2005GL024650, 2006.

Rezeau, L., Roux, A., and Russell, C. T.: Characterization of SmallScale Structures at the Magnetopause From ISEE Measurements, J. Geophys. Res., 98, 179-186, 1993.

Rodriguez, P. and Gurnett, D. A.: Electrostatic and Electromagnetic Turbulence Associated With the Earth's Bow Shock, J. Geophys. Res., 80, 19-31, 1975.

Rönnmark, K.: WHAMP — Waves in Homogeneous Anisotropic Multicomponent Plasmas, KGI Report 179, Kiruna Geophysical Institute, 1982.

Smith E. J., Holzer, R. E., McLeod, M. G., et al.: Magnetic Noise in the Magnetosheath in the Frequency Range 3-300 Hz, J. Geophys. Res., 72, 4803-4813, 1967.

Sonett, C. P., Judge, D. L., and Kelso, J. M.: Evidence concerning instabilities of the distant geomagnetic field: Pioneer 1, J. Geophys. Res., 64, 941-943, 1959.

Stenberg, G., Oscarsson, T., André, M., et al.: Electron-scale sheets of whistlers close to the magnetopause, Ann. Geophys., 23, 3715-2725, 2005,

http://www.ann-geophys.net/23/3715/2005/.

Storey, L. R. O.: An investigation of whistling atmospherics, Philosophical transactions of the Royal Society of London, 246, 113141, 1953.

Tsurutani, B. T. and Smith, E. J.: Two Types of Magnetospheric ELF Chorus and Their Substorm Dependences, J. Geophys. Res., 82, 5112-5128, 1977.

Vaivads, A., André, M., Buchert, S. C., et al.: Cluster observations of lower hybrid turbulence within thin layers at the magnetopause, Geophys. Res. Lett., 31, L03804, doi:10.1029/2003GL018142, 2004a.

Vaivads, A., Khotyaintsev, Y., André, M., et al.: Structure of the magnetic reconnection diffusion region from four-spacecraft observations, Phys. Rev. Lett., 93, 105001, doi:10.1103/PhysRevLett.93.105001, 2004b.

Vaivads, A., Santòlik, O., Stenberg, G., André, M., Owen, C. J., Canu, P., and Dunlop, M.: The source of whistler emissions 
at the dayside magnetopause, Geophys. Res. Lett., 34, L09106, doi:10.1029/2006GL029195, 2007.
Zhang Y., Matsumoto, H., and Kojima, H.: Bursts of whistler mode waves in the upstream of the bow shock: Geotail observations, J. Geophys. Res., 103, 20 529-20 540, 1998. 